\title{
Concomitant modulation of PTEN and Livin in gastric cancer treatment
}

\author{
CHUN-LIN ZHAO ${ }^{1 *}$, SHENG-NA HAN $^{2 *}$, ZHI-JU WANG ${ }^{3}$, SHU-HUI WANG ${ }^{2}$, \\ GUO-QIANG ZHAO ${ }^{3}$, XIE-FU ZHANG ${ }^{1}$ and JIA-XIANG WANG ${ }^{4}$ \\ ${ }^{1}$ Department of General Surgery, The First Affiliated Hospital of Zhengzhou University; Departments of ${ }^{2}$ Pharmacology \\ and ${ }^{3}$ Physiology, School of Basic Medical Sciences, Zhengzhou University; ${ }^{4}$ Department of Pediatric Surgery, \\ The First Affiliated Hospital of Zhengzhou University, Zhengzhou, Henan 450052, P.R. China
}

Received September 28, 2017; Accepted February 2, 2018

DOI: $10.3892 /$ ijmm.2018.3475

\begin{abstract}
Phosphatase and tensin homolog deleted on chromosome 10 (PTEN) and Livin are important in the development of gastric cancer (GC). PTEN and Livin are involved in the regulation of tumor cell proliferation, migration and apoptosis. The modulation of PTEN or Livin has been investigated extensively in various cancer models. However, no studies have been performed to evaluate the combined effect of concurrently modulating these two genes on the development of GC. In the present study, the BGC823 human gastric carcinoma cell line was transfected with a dual gene modified vector (pCL-neo-PTEN-siLivin) in parallel with single gene modified vectors (pCL-neo-PTEN or pRNAT-U6.1-siLivin), and an empty control vector. Dual gene modulation (pCL-neo-PTEN-siLivin) had a more marked effect on the inhibition of cell proliferation, induction of apoptosis, and reduction of cell penetration in Matrigel, compared with either single gene alone or empty vector transfection. In a xenograft nude mouse model, the inoculation of pCL-neo-PTEN-siLivin-transfected BGC823 cells led to a markedly reduced tumor burden, compared with that in all other inoculation groups. In conclusion, the overexpression of PTEN concomitant with Livin gene silencing was confirmed as a feasible and effective in vitro and in vivo gene modulation method, which may represent a potential therapeutic strategy for the treatment of GC.
\end{abstract}

\section{Introduction}

Gastric cancer (GC) is a malignant tumor arising from the lining of the stomach. GC is the fifth most common type of

Correspondence to: Dr Jia-Xiang Wang, Department of Pediatric Surgery, The First Affiliated Hospital of Zhengzhou University, 1 East-Jian She Road, Zhengzhou, Henan 450052, P.R. China

E-mail: jxwangzzu@126.com

*Contributed equally

Key words: Livin, phosphatase and tensin homolog deleted on chromosome 10, plasmid, gastric cancer, overexpression, silencing cancer in the world, and remains the second most common cause of cancer-associated mortality worldwide $(1,2)$. The development of GC may result from complex interactions between multiple factors, including genetic and epigenetic alterations of oncogenes and tumor-suppressor genes, cell-cycle regulators, cell adhesion molecules and DNA repair genes $(3,4)$.

Phosphatase and tensin homology deleted on chromosome $10(P T E N)$ is a tumor suppressor gene belonging to the phosphatase family, and is one of the most frequently mutated tumor suppressors in several types of human cancer (5). PTEN is important in cell proliferation, migration and apoptosis (6). In GC, a significant reduction in the expression of PTEN has been reported. In addition, the expression of PTEN negatively correlates with tumor size, Borrmann classification, lymph node metastasis and tumor staging $(7,8)$. Loss of a PTEN allele has been identified in $70 \%$ of patients with prostate cancer at the time of diagnosis (9). The inactivation of PTEN can be attributed to gene mutation, hypermethylation, microRNA-mediated regulation and post-translational phosphorylation (10). Previous studies have shown that the functional inactivation of PTEN has been detected in multiple cases of GC and other tumors (11-13). These reports suggest that the inactivation of PTEN is closely associated with the incidence, progression and prognosis of GC.

Livin, a member of the inhibitor-of-apoptosis protein (IAP) family is expressed in a variety of tumors, including GC, melanoma, neuroblastoma, mesothelioma and osteosarcoma (14-16). The expression of Livin is increased in human GC, and correlates with tumor differentiation and lymph node metastases. The underlying mechanism of its effect indicates that Livin protein binds directly to caspases, thereby inhibiting apoptosis and promoting tumor growth. By contrast, the knockdown of Livin inhibits cell growth and invasion $(17,18)$. Therefore, the results reported to date suggest that the downregulation of Livin may be a potential therapeutic strategy for GC.

PTEN and Livin are involved in the regulation of tumor cell proliferation, migration and apoptosis, in which PTEN suppresses and Livin promotes cancer development. Although the modulation of PTEN or Livin has been investigated 
extensively in various cancer models $(7,8,19)$, no studies have been performed to evaluate the combined effect on the development of GC of concurrently modulating these two genes. Therefore, the present study focused on evaluating the biological effect of this dual gene modulation on the development of GC by employing recombinant vectors with PTEN and/or Livin gene modulation capacity, previously constructed in the laboratory (20).

\section{Materials and methods}

Mice. All animal experiments were performed in accordance with protocols approved by the Animal Ethics Committee of Zhengzhou University (Zhengzhou, China). The 6-8-week-old $\mathrm{BALB} / \mathrm{c}$ male mice were purchased from the Laboratory Animal Center of Zhengzhou University. All animal experimental procedures were approved by the Institutional Animal Care and Use Committee of Zhengzhou University. The mice were bred in the animal room with continuous air circulation at $25-27^{\circ} \mathrm{C}, 40-60 \%$ humidity and a regular $12 \mathrm{~h} \mathrm{light/dark}$ cycle. Sterile food and water containing multi-vitamins was provided to the mice.

BGC823 cell transfection. The following vectors were used in the present study: pCL-neo-PTEN vector for the overexpression of PTEN; pRNAT-U6.1-siLivin vector for silencing of the Livin gene; pCL-neo-PTEN-siLivin vector for the simultaneous overexpression of PTEN and silencing of the Livin gene; empty vector as a transfection control. The vectors were constructed as described previously (20). These vectors were transfected into BGC823 cells (Culture Collection of the Chinese Academy of Sciences, Shanghai, China) using a Lipofectamine $^{\mathrm{TM}} 2000$ transfection kit (Invitrogen; Thermo Fisher Scientific, Inc., Waltham, MA, USA). The BGC823 cells were cultured in a 6-well plate in RPMI-1640 culture medium at a concentration of $1 \times 10^{6}$ cells $/ \mathrm{ml}$ at $37^{\circ} \mathrm{C}$ and in the presence of $5 \% \mathrm{CO}_{2}$. The stable transfection clones from the above transfections were selected following multiple rounds of culture and selection.

Reverse transcription-quantitative polymerase chain reaction $(R T-q P C R)$ analysis. Tumor tissue $(20-30 \mathrm{mg})$ from each mouse was ground in liquid nitrogen. Total RNA was extracted and purified using an RNA extraction kit (cat no. 15596026; Invitrogen; Thermo Fisher Scientific, Inc.). The RNA sample was then reverse transcribed into cDNA using a high capacity cDNA reverse transcription kit (cat no. RR047A; Takara Biotechnology Co., Ltd., Dalian, China). RT-qPCR analysis was performed using a real-time PCR kit (Baocheng Biotech Co., Ltd., Dalian, China). Subsequently, qPCR was performed using the SYBR Green Master mix (cat no. 4472908; Thermo Fisher Scientific, Inc.) on a 7500 Fast Real-Time PCR system (Applied Biosystems; Thermo Fisher Scientific, Inc.). Thermocycling conditions were as follows; $50^{\circ} \mathrm{C}$ for $2 \mathrm{~min}$; pre-denaturation at $95^{\circ} \mathrm{C}$ for $2 \mathrm{~min}$; followed by $15 \mathrm{sec}$ at $95^{\circ} \mathrm{C}$ then $1 \mathrm{~min}$ at $60^{\circ} \mathrm{C}$ for 40 cycles; melt curve was at $95^{\circ} \mathrm{C}$ for $15 \mathrm{sec}, 1 \mathrm{~min}$ at $60^{\circ} \mathrm{C}, 15 \mathrm{sec}$ at $95^{\circ} \mathrm{C}$ and $15 \mathrm{sec}$ at $60^{\circ} \mathrm{C}$.

RNA was normalized to the expression levels of $\beta 2$-microglobulin $(B 2 M)$ and relative expression was calculated. The sequences of the PCR primers used were as follows: B2M, forward 5'-TCCATCCGACATTGAAGTTG-3' and reverse 5'-ACACGGCAGGCATACTCAT-3'; PTEN, forward 5'-TGGCGGAACTYGCAATCC-3' and reverse 5'-GCTGAGGAACTCAAAGTAC-3'; Livin, forward 5'-TCC TGCTCCGGTCAAAAGG-3' and reverse 5'-GCTGCGTCT TCCGGTTCTT-3'. RNA levels were normalized to $\beta$-actin expression and relative expression was calculated using the $2^{-\Delta \Delta C q}$ method (21).

Western blot analysis. The cells or tumor tissues were harvested and extracted using radioimmunoprecipitation assay lysis buffer (50 mM Tris- $\mathrm{HCl}, \mathrm{pH} 7.5,150 \mathrm{mM} \mathrm{NaCl}$, $1 \%$ deoxycholate, $1 \%$ Triton X-100, 2 mM EDTA, 0.1\% SDS and $50 \mathrm{mM} \mathrm{NaF}$ ) containing a protease inhibitor cocktail (Roche Diagnostics GmbH, Mannheim, Germany). Protein concentrations were determined using the bicinchoninic acid method (Pierce; Thermo Fisher Scientific, Inc.). The extracts were heated for $5 \mathrm{~min}$ in loading buffer, and equal quantities $(40 \mu \mathrm{g})$ of cell extracts were separated on $12 \%$ SDS-PAGE gels. The separated protein bands were transferred onto polyvinylidene fluoride membranes and then blocked in $10 \%$ skim milk for $1 \mathrm{~h}$ at room temperature. Primary antibodies against PTEN (cat no. sc-133242; Santa Cruz Biotechnology, Inc., California, CA, USA), Livin (cat no. ab-127979; Abcam, Cambridge, MA, USA) and $\beta$-actin (cat no. sc-130301, Santa Cruz Biotechnology, Inc.) were diluted 1:1,000 according to the manufacturer's protocol and incubated overnight at $4^{\circ} \mathrm{C}$. The membranes were then incubated with the corresponding horseradish peroxidase-conjugated secondary antibodies (1:5,000; cat no. sc-2005; Santa Cruz Biotechnology, Inc.) at room temperature for $1 \mathrm{~h}$. Chemiluminescent solution (NEN Life Science, Boston, MA, USA) was used to visualize the blot following exposure onto hyper film (GE Healthcare Life Sciences, Pittsburgh, PA, USA) for $5 \mathrm{~min}$. Blots were analyzed and quantified by Quantity One software (4.6.2 version; Bio-Rad Laboratories, Inc., Hercules, CA, USA).

Cell proliferation assay. Cells in the exponential phase were collected and cultured in a 96-well culture plate at a concentration of $1 \times 10^{4}$ cells in a volume of $200 \mu \mathrm{l}$ per well at $37^{\circ} \mathrm{C}$ with $5 \% \mathrm{CO}_{2}$ for $1-5$ days. Each day, $20 \mu \mathrm{l}$ of 3-(4,5-dimethylthiazol-2-yl)-2,5-diphenyltetrazolium bromide (MTT) solution $(5 \mathrm{mg} / \mathrm{ml})$ was added to the culture and incubated for another $4 \mathrm{~h}$ at $37^{\circ} \mathrm{C}$. The culture medium was discarded and $150 \mu \mathrm{l}$ of DMSO was added for $10 \mathrm{~min}$ to dissolve the crystals. The absorption at $570 \mathrm{~nm}$ was detected in an automatic plate reader (Bio-Rad Laboratories, Inc.).

Terminal deoxynucleotidyl transferase dUTP nick end labeling (TUNEL) apoptosis assay. A TUNEL assay was used to detect apoptosis following transfection with different vectors. The cells were deposited onto glass slides, and cell climbing slides were produced and dried at room temperature. Following washing three times with phosphate-buffered saline (PBS), the cells on slides were fixed by adding $4 \%$ paraformaldehyde and then washed twice with PBS. The apoptotic cells were detected using a TUNEL kit (R\&D Systems, Inc., Minneapolis, MN, USA) according to the manufacturer's protocol. PBS was used as a negative control. Cells presenting 

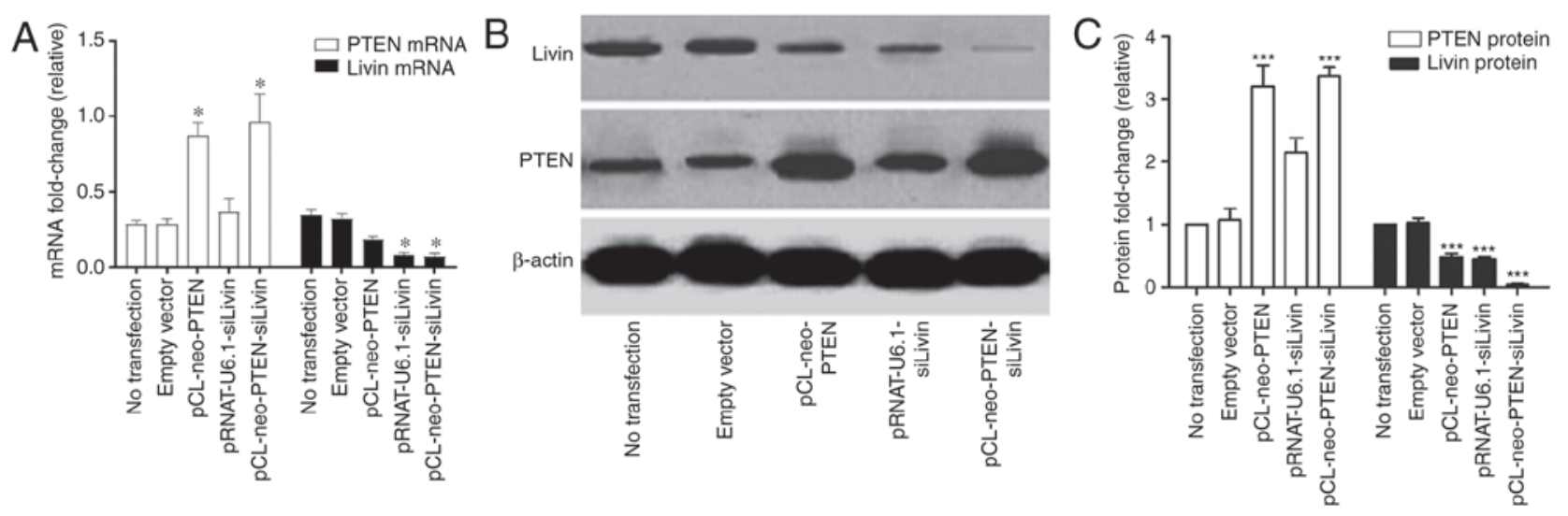

Figure 1. Characterization of PTEN and Livin gene expression in transfected BGC823 cells. BGC823 cells were transfected with vectors pCL-neo-PTEN, pRNAT-U6.1-siLivin, pCL-PTEN-siLivin, or empty control. mRNA and protein levels were evaluated by reverse transcription-quantitative polymerase chain reaction and western blot analyses, respectively. (A) mRNA levels of PTEN and Livin. 32 -microglobulin was used as an endogenous control. (B) Blots and (C) quantification of protein levels of PTEN and Livin. $\beta$-actin was used as an endogenous control. ${ }^{*} \mathrm{P}<0.05$ and ${ }^{* * * *} \mathrm{P}<0.01$ compared with the empty vector transfection group. $\mathrm{n}=6 /$ group. Data are representative of three experiments. PTEN, phosphatase and tensin homolog deleted on chromosome 10.

with brown granules in the nuclei were classified as apoptotic with an inverted microscope (X-71; Olympus Corporation, Tokyo, Japan). The percentage of apoptotic cells and the apoptotic indices were calculated by randomly counting 500 cells selected from five spots in the slides.

Detection of caspase-3 and -9 activity. Following transfection, $2 \times 10^{6} \mathrm{BGC} 823$ cells were collected, and active caspase- 3 and -9 were examined using a caspase activity detection kit (GenMed Scientifics, Wilmington, DE, USA) according to the manufacturer's protocol. The fluorescence of each sample was detected using a spectrophotometer with absorption/emission maxima of $\sim 511 / 533 \mathrm{~nm}$ (Bio-Rad Laboratories, Inc.).

Cell migration assay. In vitro cell invasion was evaluated using the Boyden Chamber Assay (Cell Biolabs, Inc., San Diego, CA, USA) as previously reported (22). Briefly, serum-free RPMI-1640 containing $3.9 \mu \mathrm{g} / \mu \mathrm{l}$ of Matrigel $^{\mathrm{TM}}$ (BD Biosciences, San Jose, CA, USA) was added to the upper chamber above the filter membrane, and incubated at $37^{\circ} \mathrm{C}$ for $2 \mathrm{~h}$. Subsequently, $200 \mu \mathrm{l}$ of serum-free cell culture supernatant from NIH3T3 cells (purchased from the Shanghai Institute of Pharmaceutical Industry, Shanghai, China) was added into the lower chamber as the chemoattractant factor. The cells $(400 \mu \mathrm{l})$ transfected with different vectors were added to the upper chamber at a concentration of $1 \times 10^{6}$ cells $/ \mathrm{ml}$ and cultured for $24 \mathrm{~h}$ at $37^{\circ} \mathrm{C}$ with $5 \% \mathrm{CO}_{2}$. The cells that had migrated into the Matrigel were detected by hematoxylin and eosin (HE) staining and those from five randomly selected spots were counted under an inverted microscope (X-71; Olympus Corporation). Five chambers were assessed for each cell line transfected by different vectors. At least three independent experiments were performed.

In vivo mouse models of GC. In vivo tumorigenesis was investigated in 6-8-week-old BALB/c nude mice (Shanghai SLAC Laboratory Animal Co. Ltd.). The 25 nude mice were divided into five groups ( $n=5 /$ group) for xenograft transplantation of non-transfected and transfected BGC823 cells. The vectors used for transfection were pCL-neo-PTEN,
pRNAT-U6.1-siLivin, pCL-neo-PTEN-siLivin, and control. Briefly, $1 \times 10^{7}(1.0 \mathrm{ml})$ BGC823 cells were inoculated subcutaneously. Tumor size was carefully measured from day 7 post-inoculation. The tumors were removed and weighed on day 28 .

Statistical analysis. Data were processed and analyzed using SPSS 13.0 (SPSS, Inc., Chicago, IL, USA). Results are shown as the mean \pm standard error of the mean. One-way analysis of variance was used to detect differences between groups. If a significant difference was found by analysis of variance, the Fisher LSD test was used to detect specific differences between the study groups. $\mathrm{P}<0.05$ was considered to indicate a statistically significant difference.

\section{Results}

Characterization of the expression of PTEN and Livin in transfected BGC823 cells. The constructs, which were previously constructed in the laboratory (pCL-neo-PTEN, pRNAT-U6.1-siLivin and pCL-neo-PTEN-siLivin) and the empty vector control were transfected into BGC823 cells. To evaluate the quality of transfection, the mRNA and protein expression levels were characterized. The results showed that the cells transfected with either pCL-neo-PTEN or pCL-neo-PTEN-siLivin vectors exhibited a significant increase in mRNA and protein expression levels of PTEN, compared with cells transfected with empty vector alone (Fig. 1A-C). In addition, a significant decrease in the mRNA and protein expression levels of Livin were observed in the cells transfected with either the pRNAT-U6.1-siLivin or pCL-neo-PTEN-siLivin vector (Fig. 1A and C). pCL-neo-PTEN transfection also resulted in a partial decrease in the mRNA and protein expression levels of Livin, although the inhibitory effect was not as pronounced as that observed in the Livin silencing groups. By contrast, transfection with the pRNAT-U6.1-siLivin vector had minimal effect on the expression of PTEN. These data suggested that PTEN may be involved in regulating the gene expression of Livin. 


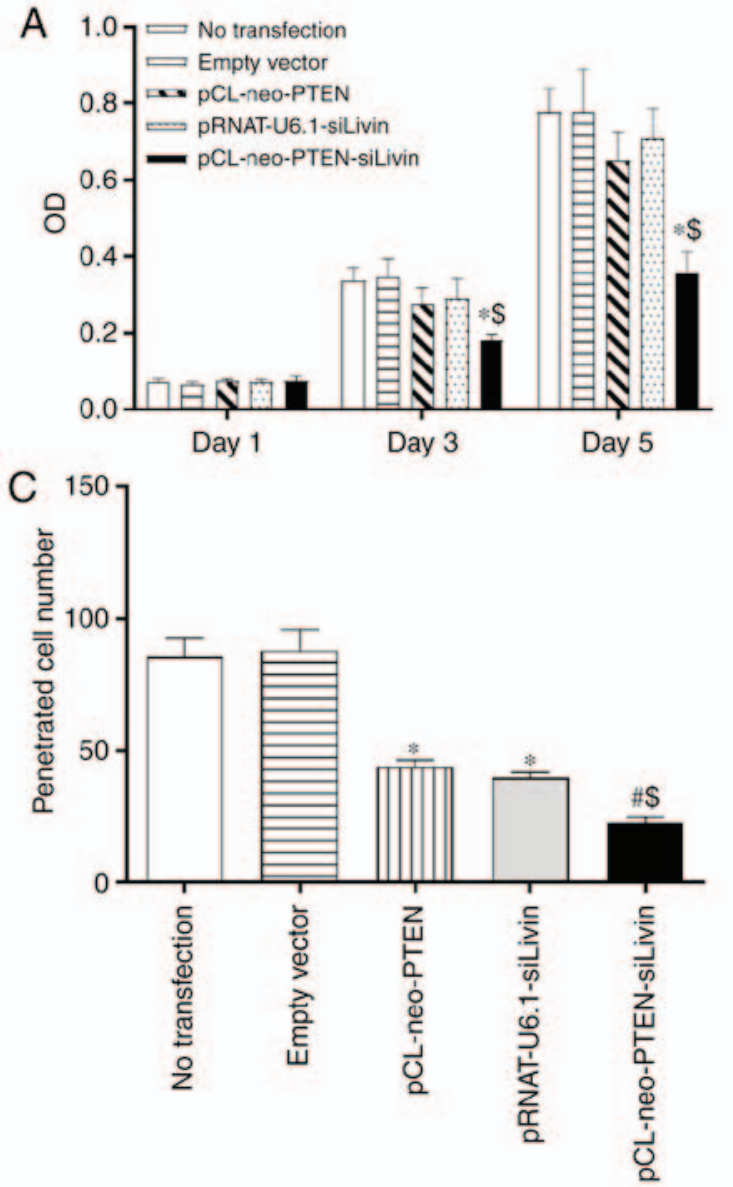

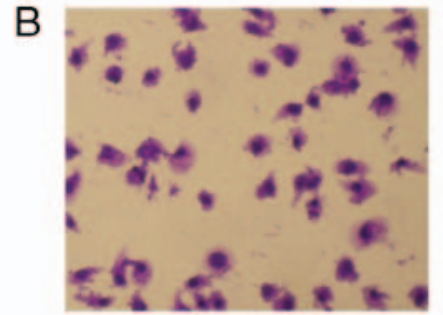

No transfection

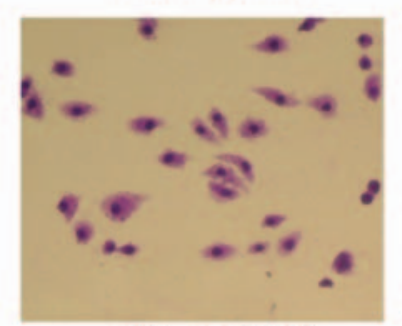

pCL-neo-PTEN
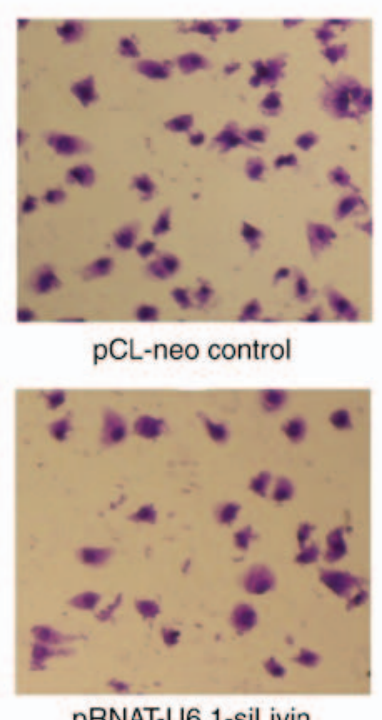

pRNAT-U6.1-siLivin

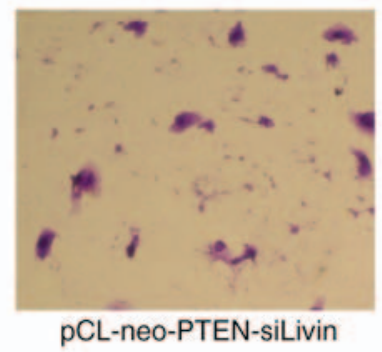

Figure 2. Characterization of the effect of modulated expression of PTEN and Livin on tumor cell proliferation and migration in vitro. (A) Proliferation of transfected BGC823 cells was measured using the 3-(4,5-dimethylthiazol-2-yl)-2,5-diphenyltetrazolium bromide assay on days 1,3 and 5. (B) Matrigel was used to characterize the migration ability of transfected BGC823 cells. Representative random spots were viewed under a microscope (magnification, x200 and $\mathrm{x} 400$ ). (C) Numbers of transfected cells penetrated through the Matrigel were analyzed. " $\mathrm{P}<0.05$ and ${ }^{\#} \mathrm{P}<0.01$, compared with empty vector transfection; ${ }^{\$} \mathrm{P}<0.05$, compared with pCL-neo-PTEN or pRNAT-U6.1-siLivin transfection. $\mathrm{n}=6$ /group. Data are representative of three experiments. PTEN, phosphatase and tensin homolog deleted on chromosome 10.

Effect of PTEN and Livin transfection on tumor cell proliferation and migration. Subsequently, the present study characterized whether the modulation of PTEN and Livin had any effect on BGC823 cell proliferation. Following transfection of the BGC823 cells with the various constructs, cell proliferation was monitored on days 1,3 , and 5 using the MTT assay. The data demonstrated that neither pCL-neo-PTEN nor pRNAT-U6.1-siLivin vector transfection alone significantly affected cell proliferation at the monitored time-points. By contrast, pCL-neo-PTEN-siLivin vector transfection, in which PTEN and Livin were modulated concurrently, significantly inhibited cell proliferation on days 3 and 5, compared with either empty vector transfection or PTEN or Livin monovector transfection $(\mathrm{P}<0.05$, Fig. $2 \mathrm{~A})$.

To evaluate whether the modulation of PTEN and Livin exerted any effect on the metastasis of transfected cells, an in vitro Matrigel assay was performed. The migrated cells were detected in the Matrigel by HE staining and the numbers of cells in five randomly selected spots were counted under the microscope. Compared with the cells transfected with the empty vector, the single (pCL-neo-PTEN or pRNAT-U6.1-siLivin transfection) and dual (pCL-neo-PTEN-siLivin transfection) gene modulation resulted in a significant inhibition of cell migration $(\mathrm{P}<0.05$ and $\mathrm{P}<0.01$ for single and dual modulation, respectively; Fig. 2B and C). Furthermore, compared with either pCL-neo-PTEN or pRNAT-U6.1-siLivin single gene transfection, dual gene modulation resulted in the maximal level of migration inhibition $(\mathrm{P}<0.05$; Fig. $2 \mathrm{~B}$ and $\mathrm{C})$. The data suggested that PTEN and Livin are critical in cell migration, and concurrent PTEN and Livin gene modulation may result in the most marked inhibition of tumor cell migration.

Modulation of PTEN and Livin induces apoptosis by activating the caspase-signaling pathway. The present study used TUNEL staining to evaluate whether modulation of the PTEN and Livin genes affected apoptosis. Single (pCL-neo-PTEN or pRNAT-U6.1-siLivin transfection) and dual (pCL-neo-PTEN-siLivin transfection) gene modulation resulted in a significant increase in the percentage of apoptotic cells, compared with that in the control (Fig. 3A). The percentages of apoptotic cells observed following pCL-neo-PTEN, pRNAT-U6.1-siLivin, and pCL-neo-PTEN-siLivin transfections were $8.67 \pm 1.27,10.39 \pm 1.31$ and $16.72 \pm 1.84$, respectively. Furthermore, compared with either pCL-neo-PTEN or pRNAT-U6.1-siLivin single gene transfection, dual gene 

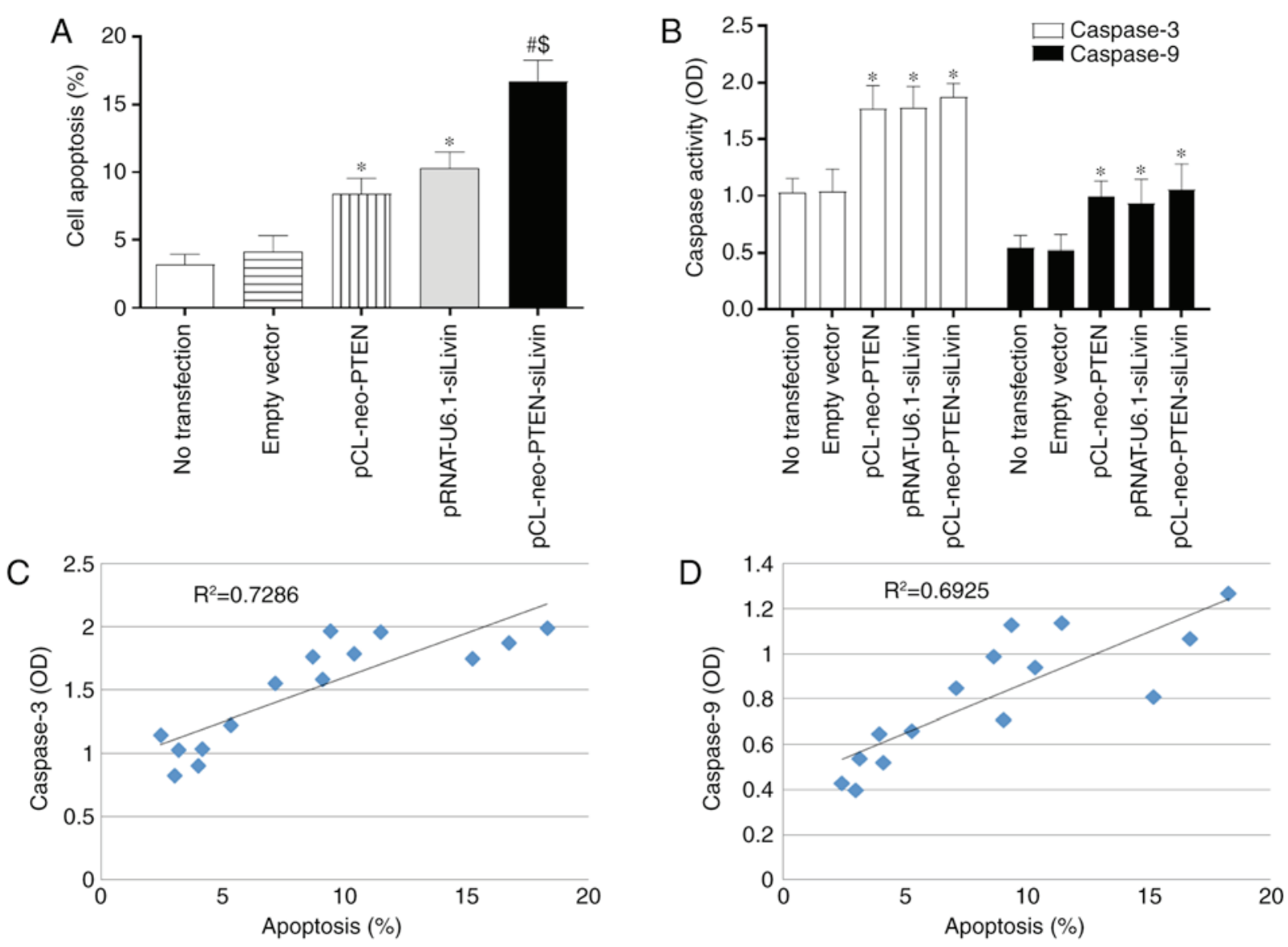

Figure 3. Modulation of the expression of PTEN and Livin induces apoptosis by activating the caspase-signaling pathway. (A) Terminal deoxynucleotidyl transferase dUTP nick end labeling staining was used to assess the level of apoptosis in transfected BGC823 cells. (B) Caspase-3 and -9 activity was determined using a caspase activity detection kit. (C) Correlation between apoptosis and caspase-3 activity. (D) Correlation between apoptosis and caspase-9 activity. ${ }^{*} \mathrm{P}<0.05$ and ${ }^{\#} \mathrm{P}<0.01$, compared with empty control vector transfection; ${ }^{\$} \mathrm{P}<0.05$, compared with pCL-neo-PTEN or pRNAT-U6.1-siLivin transfection. $\mathrm{n}=6$ /group. Data are representative of three experiments. PTEN, phosphatase and tensin homolog deleted on chromosome 10 .

modulation resulted in the highest level of cell apoptosis (P<0.05; Fig. 3A).

Caspase activity is a marker of apoptosis. Therefore, the present study examined the activities of caspase-3 and caspase- 9 in the transfected BGC823 cells. Single (pCL-neo-PTEN or pRNAT-U6.1-siLivin transfection) and dual (pCL-neo-PTEN-siLivin transfection) gene modulation induced significant increases in caspase-3 and caspase- 9 activities $(\mathrm{P}<0.05$; Fig. 3B). However, there were no significant differences between the three transfection groups. Further examination revealed that the observed apoptosis was positively correlated with caspase 3 and caspase 9 activity, $\left(\mathrm{R}^{2}=0.7286\right.$ and $\mathrm{R}^{2}=0.6925$, respectively; Fig. $3 \mathrm{C}$ and $\left.\mathrm{D}\right)$. These data suggested that the modulation of PTEN and Livin induced apoptosis via activation of the caspase signaling pathway.

Modulation of PTEN and Livin inhibits tumor growth in vivo. To determine the effect of PTEN and Livin gene modulation on tumor growth in vivo, various vector-transfected BGC823 cells were injected into nude mice. Tumor growth was monitored weekly between days 0 and 28 , when the mice were sacrificed. The tumors grew continuously from day 0 to day 28 in the non-transfected and empty vector transfection groups (Fig. 4A). By contrast, tumor growth was suppressed in the single gene (pCL-neo-PTEN or pRNAT-U6.1-siLivin) and dual gene (pCL-neo-PTEN-siLivin) transfected groups, with the dual gene modulation group demonstrating the most marked suppression of tumor growth (Fig. 4A). The percentage tumor growth inhibition (\% TGI) in the pCL-neo-PTEN, pRNAT-U6.1-siLivin and pCL-neo-PTEN-siLivin transfection groups was 45\%, 51 and $72 \%$, respectively (Fig. 4B).

Characterization of the gene expression of PTEN and Livin in tumors. To verify whether the observed inhibitory effects on tumor growth were specific to PTEN or Livin gene modulation, the expression levels of PTEN and Livin in the tumor tissues were examined. The mice were sacrificed 28 days following establishment of the xenograft model and the tumors were collected. Tumor tissues from the mice with cells expressing pCL-neo-PTEN or pCL-neo-PTEN-siLivin expressed significantly higher mRNA and protein levels of PTEN, compared with those with control or empty vector cells $(\mathrm{P}<0.05$; Fig. 5A-C). Tumor tissues from mice with cells expressing pRNAT-U6.1-siLivin or pCL-neo-PTEN-siLivin expressed significantly lower mRNA and protein levels of Livin, compared with those with control or empty vector cells $(\mathrm{P}<0.05$; Fig. 5A-C). These data indicated that the transfected cells maintained stable expression of the target genes in the tumors in vivo. These results showed that the in vivo 

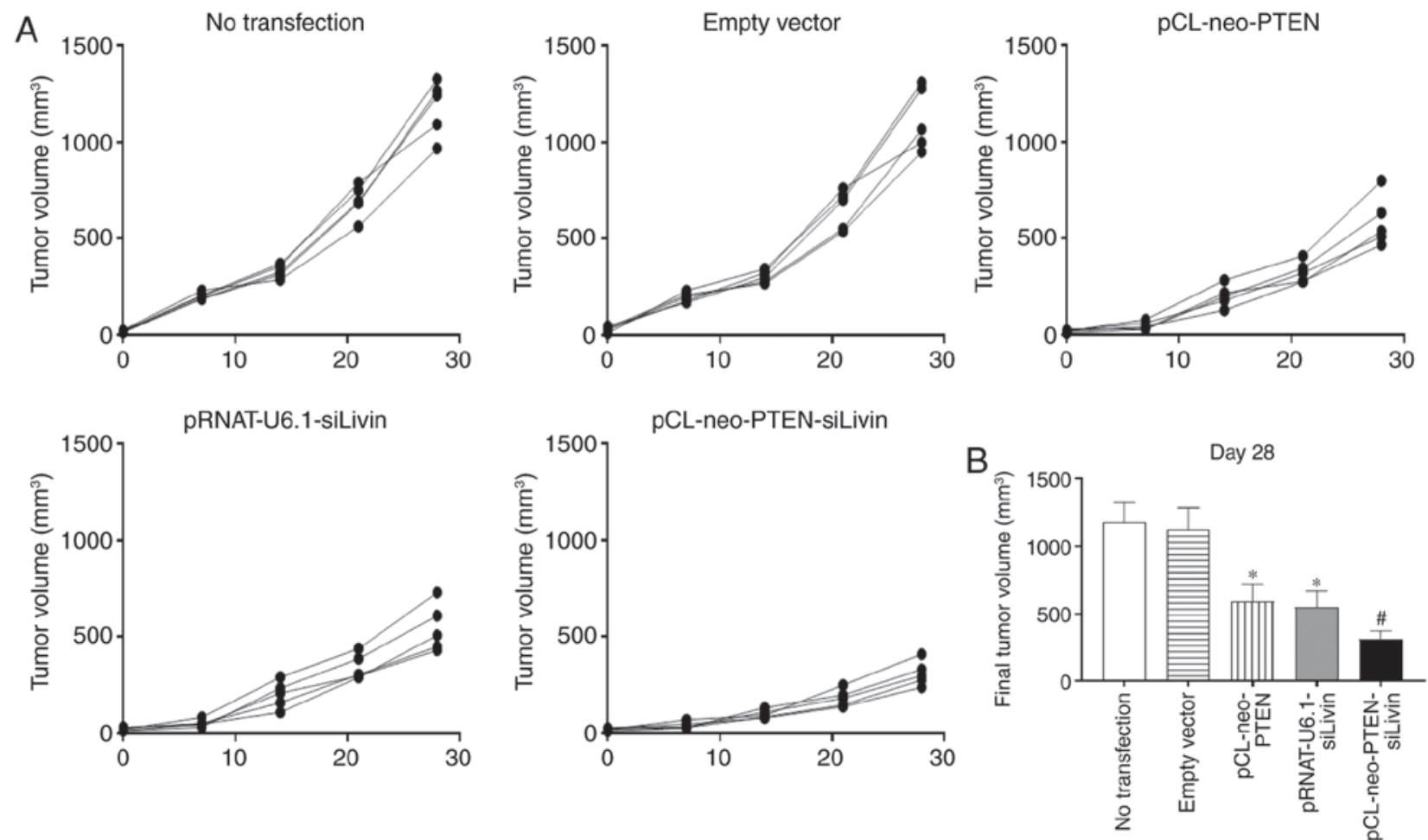

Figure 4. Modulation of the gene expression of PTEN and Livin inhibits tumor growth in vivo. Transfected BGC823 cells were inoculated subcutaneously into nude mice and tumor growth was monitored weekly. (A) Tumor volume was measured weekly between day 0 and 28. (B) The volume of tumors from each group was measured on day $28 .{ }^{~} \mathrm{P}<0.05$ and ${ }^{\#} \mathrm{P}<0.01$ compared with $\mathrm{pCL}$-neo control vector transfection. $\mathrm{n}=5 /$ group. PTEN, phosphatase and tensin homolog deleted on chromosome 10 .
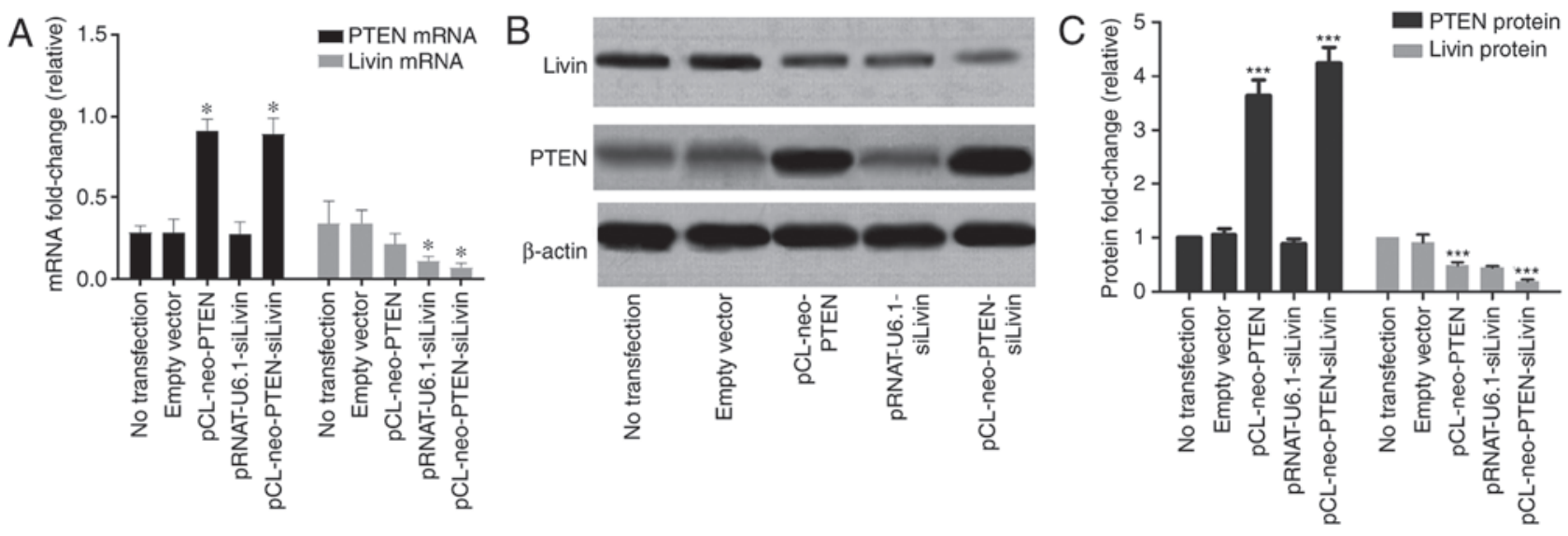

Figure 5. Characterization of the expression of PTEN and Livin in tumors. Tumor tissues were excised and total RNA and protein were measured. (A) mRNA expression of PTEN and Livin was detected by reverse transcription-quantitative polymerase chain reaction analysis, with $\beta 2$-microglobulin as an internal control. (B) Blots and (C) quantification of PTEN and Livin proteins detected by western blot analysis, with $\beta$-actin as a control. ${ }^{*} \mathrm{P}<0.05$ and ${ }^{* * * *} \mathrm{P}<0.01$ compared with the empty vector transfection group. $n=5 /$ group. PTEN, phosphatase and tensin homolog deleted on chromosome 10 .

experiments on tumor tissues and in vitro experiments using BGC823 cells produced comparable results.

\section{Discussion}

Several studies have demonstrated an association between the expression of PTEN and Livin and the malignancies of renal cell carcinoma (23,24), breast cancer (25) and retinocytoma (26). Specifically, a low expression of PTEN and high expression of Livin were significantly associated with the clinical stage and lymph node metastases of patient malignancies. However, no studies have investigated a direct correlation between PTEN and Livin gene expression and the malignancy of GC.

In the present study, it was shown that the overexpression of PTEN or gene silencing of Livin promoted apoptosis in BGC823 cells and inhibited cell proliferation. The data demonstrated that the combined effect of PTEN/Livin dual gene modulation on apoptosis and proliferation was more marked, compared with that of either single gene alone. The functional effects of PTEN and Livin gene regulation on GC apoptosis and proliferation were further supported by the xenograft experiments in nude mice. The results suggested that the 
overexpression of PTEN concomitant with the gene silencing of Livin may represent a potential therapeutic strategy for gene therapy in the treatment of GC.

Caspase-3 is a proteinase with a crucial role in the apoptotic pathway. Caspase-3 can be detected in almost all cell types, emphasizing its importance in modulating cell survival and death (23). The increased expression of PTEN in cultured neonatal rat primary cardiomyocytes leading to increased caspase-3 activity and apoptosis has been reported (24), which suggests that caspase- 3 is the major effector of PTEN. PTEN has been shown to inhibit cell proliferation and apoptotic function by downregulating the AKT/PKB pathway (25). By contrast, Livin demonstrates anti-apoptotic activity. Livin contains a unique Baculovirus IAP repeat (BIR) domain and a Really Interesting New Gene (RING) finger motif domain (26). The BIR domain forms a novel zinc-fold, which is the critical motif for the anti-apoptotic activity of the parent protein, its interaction with caspase 3, -7 and -9 , and its E3 ubiquitin ligase (27). The RING domain is critical in tumor necrosis factor- $\alpha$-mediated nuclear factor (NF)- $\kappa \beta$ activation, thereby providing an additional mechanism for the anti-apoptotic activity of Livin (28). Livin also promotes the degradation of the inhibitor of apoptosis antagonist SMAC/DIABLO $(29,30)$. These data suggest that PTEN and Livin exert opposite effects on the caspase signaling pathway. Consistent with this, the present study observed significantly increased caspase-3 and caspase- 9 activity in BGC823 cells overexpressing PTEN, with silenced Livin gene expression, or with dual modulation. Therefore, the results are consistent with those previously published and showed that the PTEN and Livin genes are important in apoptosis by regulating caspase-3/9 activity. Although dual gene modulation led to the most marked effect on apoptosis, no significant difference in caspase activity was identified between the three transfection groups (Fig. 3B). This suggests that additional mechanisms, including Fas/Fasl signaling and cytochrome $c$ release, may trigger cell death. Further investigations are warranted to address which signaling cascades are involved and the underlying mechanisms through which they are contributing to apoptosis in BGC823 cells.

PTEN is important in the regulation of tumor cell metastasis. Hwang et al showed that PTEN enhanced tumor metastasis through vascular endothelial factor and matrix metalloproteinases (31). In other studies, the overexpression of PTEN inhibited glioblastoma cell migration (32), and PTEN-knockdown enhanced cell migration in fibroblasts by regulating FAK, a cytoplasmic phosphoprotein activated by integrin (33). Livin was shown to regulate tumor cell invasion, the first step of metastasis, through the $N F-\kappa \beta$ signaling pathway $(34,35)$. Furthermore, knockdown of the Livin gene inhibited tumor invasion by inhibiting the mitogen-activated protein kinase (MAPK) signaling $(17,36)$. In the present study, the migration of BGC823 cells was significantly inhibited following transfection with a vector overexpressing PTEN, silencing Livin, or modulating the two genes, with dual transfection having the highest inhibitory effect. Further experiments are required to characterize whether molecules, including FAK, NF- $\kappa \beta$ and MAPK, are involved in metastasis in GC; however, they are presently beyond the scope of the present study.
The results of the present study are consistent with those of previous reports showing that either the overexpression of PTEN or silencing of the Livin gene significantly inhibited cell proliferation and invasion, and induced apoptosis in GC. The present study successfully established in vitro and in vivo models with the simultaneous overexpression of PTEN and silencing of Livin for the first time, to the best of our knowledge. The results demonstrated that dual gene modulation produced more marked antitumor and antimetastatic effects, compared with either single gene modulation alone, in vitro and in vivo. Therefore, the simultaneous overexpression of PTEN and silencing of Livin may represent a novel therapeutic approach for the treatment of GC.

\section{Acknowledgements}

This study was supported by the Major Public Interest Foundation of Henan Province, China (grant no. HNZB2010N91).

\section{Competing interests}

The authors declare that they have no competing interests.

\section{References}

1. Guggenheim DE and Shah MA: Gastric cancer epidemiology and risk factors. J Surg Oncol 107: 230-236, 2013.

2. Yeoh KG: How do we improve outcomes for gastric cancer? J Gastroenterol Hepatol 22: 970-972, 2007.

3. Tan IB, Ng I, Tai WM and Tan P: Understanding the genetic basis of gastric cancer: Recent advances. Expert Rev Gastroenterol Hepatol 6: 335-341, 2012.

4. Nagini S: Carcinoma of the stomach: A review of epidemiology, pathogenesis, molecular genetics and chemoprevention. World J Gastrointest Oncol 4: 156-169, 2012.

5. Stambolic V, Suzuki A, de la Pompa JL, Brothers GM, Mirtsos C, Sasaki T, Ruland J, Penninger JM, Siderovski DP and Mak TW: Negative regulation of PKB/Akt-dependent cell survival by the tumor suppressor PTEN. Cell 95: 29-39, 1998.

6. She QB, Solit DB, Ye Q, O'Reilly KE, Lobo J and Rosen N: The BAD protein integrates survival signaling by EGFR/MAPK and PI3K/Akt kinase pathways in PTEN-deficient tumor cells. Cancer Cell 8: 287-297, 2005.

7. Zhang LL, Liu J, Lei S, Zhang J, Zhou W and Yu HG: PTEN inhibits the invasion and metastasis of gastric cancer via downregulation of FAK expression. Cell Signal 26: 1011-1020, 2014.

8. Katoh M: WNT/PCP signaling pathway and human cancer (review). Oncol Rep 14: 1583-1588, 2005.

9. Chen Z, Trotman LC, Shaffer D, Lin HK, Dotan ZA, Niki M, Koutcher JA, Scher HI, Ludwig T, Gerald W, et al: Crucial role of p53-dependent cellular senescence in suppression of Pten-deficient tumorigenesis. Nature 436: 725-730, 2005.

10. Xu WT, Yang Z and Lu NH: Roles of PTEN (Phosphatase and Tensin Homolog) in gastric cancer development and progression. Asian Pac J Cancer Prev 15: 17-24, 2014.

11. Koike H, Nozawa M, De Velasco MA, Kura Y, Ando N, Fukushima E, Yamamoto Y, Hatanaka Y, Yoshikawa K, Nishio K and Uemura H: Conditional PTEN-deficient mice as a prostate cancer chemoprevention model. Asian Pac J Cancer Prev 16: 1827-1831, 2015.

12. Kechagioglou P, Papi RM, Provatopoulou X, Kalogera E, Papadimitriou E, Grigoropoulos P, Nonni A, Zografos G, Kyriakidis DA and Gounaris A: Tumor suppressor PTEN in breast cancer: Heterozygosity, mutations and protein expression. Anticancer Res 34: 1387-1400, 2014.

13. Nakanishi A, Kitagishi Y, Ogura Y and Matsuda S: The tumor suppressor PTEN interacts with p53 in hereditary cancer (Review). Int J Oncol 44: 1813-1819, 2014. 
14. Kim DK, Alvarado CS, Abramowsky CR, Gu L, Zhou M, Soe MM, Sullivan K, George B, Schemankewitz E and Findley HW: Expression of inhibitor-of-apoptosis protein (IAP) livin by neuroblastoma cells: Correlation with prognostic factors and outcome. Pediatr Dev Pathol 8: 621-629, 2005.

15. Kleinberg L, Lie AK, Florenes VA, Nesland JM and Davidson B: Expression of inhibitor-of-apoptosis protein family members in malignant mesothelioma. Hum Pathol 38: 986-994, 2007.

16. Li CJ, Cong Y, Liu XZ, Zhou X, Shi X, Wu SJ, Zhou GX and $\mathrm{Lu}$ M: Research progress on the livin gene and osteosarcomas. Asian Pac J Cancer Prev 15: 8577-8579, 2014.

17. Ou JM, Ye B, Qiu MK, Dai YX, Dong Q, Shen J, Dong P, Wang XF, Liu YB, Quan ZW and Fei ZW: Knockdown of Livin inhibits growth and invasion of gastric cancer cells through blokkade of the MAPK pathway in vitro and in vivo. Int J Oncol 44 276-284, 2014.

18. Chung CY, Park YL, Kim N, Park HC, Park HB, Myung DS, Kim JS, Cho SB, Lee WS and Joo YE: Expression and prognostic significance of Livin in gastric cancer. Oncol Rep 30: 2520-2528, 2013.

19. Crnković-Mertens I, Wagener N, Semzow J, Gröne EF, Haferkamp A, Hohenfellner M, Butz K and Hoppe-Seyler F: Targeted inhibition of Livin resensitizes renal cancer cells towards apoptosis. Cell Mol Life Sci 64: 1137-1144, 2007.

20. Zhao CL, Wang JX, Zhang XF, Zhao GQ and Wang ZJ Construction and identification of gene vector expressing PTEN while simultaneously silencing Livin. Zhonghua Yi Xue Za Zhi 90: 2428-2432, 2010 (In Chinese)

21. Livak KJ and Schmittgen TD: Analysis of relative gene expression data using real-time quantitative PCR and the 2(-Delta Delta C(T)) method. Methods 25: 402-408, 2001.

22. Hwang TL, Changchien TT, Wang CC and Wu CM: Claudin-4 expression in gastric cancer cells enhances the invasion and is associated with the increased level of matrix metalloproteinase-2 and -9 expression. Oncol Lett 8: 1367-1371, 2014.

23. Krajewska M, Wang HG, Krajewski S, Zapata JM, Shabaik A, Gascoyne R and Reed JC: Immunohistochemical analysis of in vivo patterns of expression of CPP32 (Caspase-3), a cell death protease. Cancer Res 57: 1605-1613, 1997.

24. Schwartzbauer G and Robbins J: The tumor suppressor gene PTEN can regulate cardiac hypertrophy and survival. J Biol Chem 276: 35786-35793, 2001.
25. Sun $\mathrm{CH}$, Chang $\mathrm{YH}$ and Pan CC: Activation of the $\mathrm{PI} 3 \mathrm{~K} / \mathrm{Akt} / \mathrm{mTOR}$ pathway correlates with tumour progression and reduced survival in patients with urothelial carcinoma of the urinary bladder. Histopathology 58: 1054-1063, 2011.

26. Kasof GM and Gomes BC: Livin, a novel inhibitor of apoptosis protein family member. J Biol Chem 276: 3238-3246, 2001.

27. Hinds MG, Norton RS, Vaux DL and Day CL: Solution structure of a baculoviral inhibitor of apoptosis (IAP) repeat. Nat Struct Biol 6: 648-651, 1999.

28. Chu ZL, McKinsey TA, Liu L, Gentry JJ, Malim MH and Ballard DW: Suppression of tumor necrosis factor-induced cell death by inhibitor of apoptosis c-IAP2 is under NF-kappaB control. Proc Natl Acad Sci USA 94: 10057-10062, 1997.

29. Chang H and Schimmer AD: Livin/melanoma inhibitor of apoptosis protein as a potential therapeutic target for the treatment of malignancy. Mol Cancer Ther 6: 24-30, 2007.

30. Ma L, Huang Y, Song Z, Feng S, Tian X, Du W, Qiu X, Heese K and $\mathrm{Wu} \mathrm{M}$ : Livin promotes Smac/DIABLO degradation by ubiquitin-proteasome pathway. Cell Death Differ 13: 2079-2088, 2006.

31. Hwang PH, Yi HK, Kim DS, Nam SY, Kim JS and Lee DY: Suppression of tumorigenicity and metastasis in B16F10 cells by PTEN/MMAC1/TEP1 gene. Cancer Lett 172: 83-91, 2001.

32. Leslie NR: PTEN: An intercellular peacekeeper? Sci Signal 5: pe50, 2012

33. Maehama T, Taylor GS and Dixon JE: PTEN and myotubularin: novel phosphoinositide phosphatases. Annu Rev Biochem 70: 247-279, 2001.

34. Chen F, Yang D, Che X, Wang J, Li X, Zhang Z, Chen X and Song X: Livin mediates tumor cell invasion in the DU-145 cell line via NF-кB. Oncol Rep 27: 2010-2016, 2012.

35. Chen F, Yang D, Wang S, Che X, Wang J, Li X, Zhang Z, Chen X and Song $X$ : Livin regulates prostate cancer cell invasion by impacting the NF- $\kappa \mathrm{B}$ signaling pathway and the expression of FN and CXCR4. IUBMB Life 64: 274-283, 2012.

36. Yoon TM, Kim SA, Lee DH, Lee JK, Park YL, Lee KH, Chung IJ, Joo YE and Lim SC: Expression of Livin and the inhibition of tumor progression by Livin silencing in laryngohypopharyngeal cancer. In Vivo 28: 751-759, 2014. 